\title{
Operation Safety Risk Control Strategy of Electric Power Dispatch Automation System
}

\author{
Haijiang Wu \\ Guangdong Power Grid Company and Foshan Powder Supply Bureau, Foshan, Guangdong, 528000, China \\ whj3310@163.com
}

Keywords: Automatic Operation, Power Dispatch, Risk Control

\begin{abstract}
Power transfer is an important part of power engineering project, which is related to the effect of power energy use. With the development of self-information technology, the level of power dispatching automation in China has been improved continuously, but the failure of systematic operation will lead to safety risk problems. Strengthening the operation safety risk control of the power dispatching automation system can ensure the stable operation of the power system. This paper will study the three aspects of remote communication technology management, remote control technology management and telemetry technology management in order to promote the development of power dispatching automation in China..
\end{abstract}

\section{Introduction}

Power energy is the core energy source of our country, which is related to the development of industry, agriculture and service industry. People's daily life Related[1]. Power dispatching is the main part of power project management in China, which affects the distribution of power energy resources and the safety of power system operation. The power dispatching automation system can provide convenience for power dispatching and meet the demand of power dispatching control. Based on this, it is necessary to control the safety risk of power dispatching automation system.

\section{Remote Control Technology Management}

\subsection{Implementation Process}

The implementation of the remote control process mainly depends on the monitoring workstation SCADA (main station) man-machine interface, select the corresponding equipment to execute the process, and start the operation by remote control. The process execution command is mainly issued by the front system, and through two programs (telecontrol channel, plant station telecontrol host) to the predetermined measurement and control device to execute the remote control command. The principle of "selecting-checking-after-executing" is adopted in the execution of the command of the measurement and control device[2]. Normally, the substation has no one on duty and needs to ensure that the remote control operation is $100 \%$ successful. However, in the process of practice, due to the influence of many objective factors will lead to the failure of remote command execution, resulting in the failure of returning to school and affecting the operation of the power grid.

\subsection{Cause Analysis}

There are three main phenomena of remote control failure:(1) failure of returning to school;(2) overtime of returning to school; and (3) failure of execution.

In the face of three kinds of remote control failure, the following inspection flow can be used:(1) whether the remote control parameters of the switch are filled in abnormal;(2) whether the operation channel is normal;(3) check whether the position of the local switch switch (remote) is normal;(4) check whether the main engine (remote) is normal;(5) check whether the measurement and control device is normal; and (6) check whether the power supply is normal. There are seven reasons for the failure phenomenon, as shown in Table 1. 
Table 1. Reason for failure of remote control

\begin{tabular}{|c|c|}
\hline Cause of failure & Phenomenon \\
\hline Parameter reasons & Failure to return to school / Failure to execute \\
\hline Channel reasons & $\begin{array}{l}\text { Back-to-school failure / back-to-school timeout / } \\
\text { execution failure }\end{array}$ \\
\hline Local location & Failure to return to school / Failure to execute \\
\hline $\begin{array}{l}\text { Pressure Control } \\
\text { Problem }\end{array}$ & Failed execution \\
\hline Equipment status & $\begin{array}{c}\text { Failed execution } \\
\end{array}$ \\
\hline $\begin{array}{c}\text { Relay action } \\
\text { abnormal }\end{array}$ & $\begin{array}{l}\text { Back-to-school failure / back-to-school timeout / } \\
\text { execution failure }\end{array}$ \\
\hline Power off & Failure to return to school / Failure to execute \\
\hline
\end{tabular}

First, the parameter reason:(1) the main station parameter is not consistent with the parameter set by the teletransmission, which will lead to the above failure phenomenon;(2) the problem of setting the remote control point will lead to the problem of the back-to-school path and even the phenomenon of false remote control. Therefore, in the process of parameter setting, the consistency of the operation site parameters with the main station parameters should be maintained, and other staff members should not be required to adjust the parameters at will, even if necessary to change the parameters, but also need to be based on the value sheet for data management.

Second, channel reasons:(1) the channel under abnormal factors will affect the operation command propagation path, resulting in remote control failure phenomenon;(2) poor contact with the cable that constitutes the channel or other external interference, will lead to the operation command error code phenomenon. Too high bit error rate will cause command source code missing or broken, command source code cannot be correctly executed, such as back-to-school timeout, failure, execution failure are due to this reason[3].

Third, local position: the abnormal position of the local operation handle will lead to the obstruction of the circuit, so that the remote control in the process of returning to school failure. Therefore, in the actual work process, we need to understand the body operation mechanism box, and pay attention to the local operation handle position, timely adjustment to ensure the normal operation of the program.

Fourth, the control of pressure plate problem: if the measurement and control screen on the control plate into the working state, will lead to equipment execution failure.

Fifth, device status: when the device is locked, the transmission of information will be interrupted. For example: circuit breaker equipment circuit breakage, spring without energy storage will lead to command execution failure.

Sixth, the relay action is abnormal:(1) the relay is damaged;(2) there is a problem with the power supply of the execution relay.

Seventh, the power supply is disconnected: when the remote control power supply is in a nonworking state, it will cause the remote control point to not work properly and be in a state of power loss.

\section{Remote Communication Technology Management}

\subsection{Technical Analysis}

Remote signal is one of the signals to ensure the normal operation of power grid dispatching, which can truly reflect the operation of various power system equipment. The remote signal mainly includes the warning signal, the switch signal, the action protection and so on, needs to be transmitted through the long distance, is transported to the main station end. In fact, the state of remote communication and the data of specific parameters are the important basis for the automatic operation of the system. When the remote signal is lost, the signal is not timely and the accuracy is not high, it will cause damage to the system and threaten the security of the power grid. It should be 
noted that when there is an accident in the grid, it is necessary to control the remote signal accurately to avoid affecting the accident handling effect and the safety of the dispatcher. Therefore, in the process of remote signal management, we should try our best to reduce the problems of remote signal loss, leakage and false transmission.

\subsection{Cause Analysis}

There are many reasons for the remote signal to be in abnormal working state, which are divided into the following five cases, as shown in Table.2.

Table 2 Analysis of remote communication technology

\begin{tabular}{|c|c|}
\hline Projects & Reasons \\
\hline $\begin{array}{c}\text { Abnormal measurement and } \\
\text { control device }\end{array}$ & Failure of measurement and control device / abnormal working condition \\
\hline Electromagnetic interference & Electromagnetic interference disturbs transmission signals \\
\hline Auxiliary contact jitter & $\begin{array}{c}\text { There is a gap in the mechanical transmission / oxidation of the auxiliary } \\
\text { contacts in the external environment }\end{array}$ \\
\hline Higher BER & Channel disruption \\
\hline Handling errors & Master screen processing error / database work exception \\
\hline
\end{tabular}

First, the measurement and control device is abnormal. When the measurement and control device failure will lead to the remote signal leakage, false transmission problems, such as the movement of the main engine failure, measurement and control board failure will lead to remote signal problems. When the measurement and control device is in the abnormal working state, it will cause the contact point to carry on the signal warning, and upload the abnormal signal in time through the signal transmission path to prevent the system failure problem.

Second, electromagnetic interference problem. Electromagnetic interference will disturb the transmission signal, so that the transmission of remote signal data signal distortion changes. In general, the transmission channel (cable) is relatively long, and it is very likely to pass through the radiation area of the high-voltage equipment, which leads to the change of the signal during the circuit process, and the accuracy of the signal transmission cannot be guaranteed when the number of interference increases or the intensity increases[4]. In addition, in the process of measurement and control installation generally adopt the principle of local installation, with the help of communication optical cable interconnection transmission of information. In order to reduce the influence of external factors on the transmission cable, the special cable can be shielded by signal, and the method of time-delay retest can be used to measure the signal condition of remote signal on the operation software.

Third, auxiliary contact jitter. In general, circuit breakers and other remote signals arise from the auxiliary contacts of the operating mechanism, but when there is a gap in the mechanical transmission part, the jitter of the auxiliary contacts will occur[5]。 In addition, the oxidation of auxiliary contacts in the external environment will also lead to poor contact and lead to errors in remote signals. To solve such problems, the same method of time-lapse retest can be used to eliminate abnormal shaking of auxiliary contacts.

Fourth, the error code is higher. Higher BER will lead to the failure of receiving remote signal, which is related to channel interrupt.

Fifth, deal with mistakes. When the master screen processing error and database work abnormal will cause the remote point signal is not equal, the signal is also blocked. Staff should be careful in the maintenance process, check whether the main station work is abnormal, and timely transmission test to verify the operation of the equipment system. At the same time, the staff should conduct regular database checks to check whether the SOE event is consistent with the recorded information and avoid missing the incident. 


\section{Telemetry Technology Management}

Telemetry technology is mainly used in the monitoring of power system operation, in which telemetry is the basic content.

\subsection{Acquisition Process}

Power acquisition: acquisition of active and reactive power information from branch current, bus voltage and branch in the system. Non-electric acquisition: work around oil temperature and temperature, as shown in Table 3[6].

Table 3 Telemetry technology management acquisition process

\begin{tabular}{|c|c|}
\hline Projects & Content \\
\hline $\begin{array}{c}\text { Electricity } \\
\text { Collection }\end{array}$ & $\begin{array}{c}\text { Obtain active and reactive power information from branch current, bus voltage and } \\
\text { branch in the system }\end{array}$ \\
\hline $\begin{array}{c}\text { Non-electric } \\
\text { acquisition }\end{array}$ & Working around oil temperature and temperature \\
\hline
\end{tabular}

\subsection{Cause Analysis}

(1) Incorrect power: When the line phase sequence of the measurement and control equipment is wrong, it can be electrified, but it will lead to incorrect line power. (2) Incorrect telemetry: It is mainly due to incorrect point numbers, telemetry coefficients, etc. The staff must test the measurement data information to ensure the correctness of the data information. (3) The submeasure value does not match: when the measurement and control device is abnormal, it will lead to abnormal current and voltage, and the input terminal measurement value is not in accordance with the expected results. The staff can remove the equipment and use the high precision measuring source measuring device measuring unit. After adopting this method, it still does not accord with each other, indicating that the internal wiring of the channel or device is abnormal, and it is necessary to check the specification parameters, telecontrol mainframe and channel.

\section{Conclusion}

The power dispatching work is related to the stability of the power system operation, in the process of monitoring the dispatching automation system, the risk control of the power equipment is needed to ensure the safety and reliability of the system operation. This paper discusses the safety risk control and operation management measures from three aspects of tele-communication technology management, remote control technology management and telemetry technology management, but in the actual work process, the staff also need to combine the actual problems in the power system dispatching process to adjust, give full play to the role of safety risk control and operation management.

\section{References}

[1] Jiang, Huilan. Operation management and safety risk control of power dispatching automation system. Technological Products, no. 2, pp. 110, 2017.

[2] Huang, Hanhua., Jiang, Ye., Chen, Bingwei. Risk early warning mechanism in power communication network maintenance mode. Computer Science and Applications, vol. 7, no. 03, pp. 262-273, 2017.

[3] Zhang, Wenpeng. Risk analysis of safe operation of grid dispatching automation system. China Chemical Trade, vol. 10, no. 34, pp. 194, 2018.

[4] Hou, Junfeng. Line safety operation analysis in power dispatching work. Construction Engineering Technology and Design, no. 23, pp. 2295, 2018. 
[5]Sun, Xiao. Integrated monitoring and intelligence of grid dispatching automation. Construction Engineering Technology and Design, no. 15, pp. 206, 2018.

[6] Nie, Xiaoyu., Lu, Peng. Power dispatch operation safety risk and prevention strategy. Digital Design (D), no. 8, pp. 185-186, 2018. 